Finisterra, XXXVI, 71, 2001, pp. 103-113

\title{
ANÁLISIS DE LA ACIDEZ DE LA PRECIPITACIÓN EN LA PENÍNSULA IBÉRICA
}

\author{
E. MARÍN ${ }^{1}$ \\ N. LAVÍN ${ }^{2}$ \\ E. HERNÁNDEZ ${ }^{1}$ \\ A. RÚA ${ }^{3}$
}

\begin{abstract}
Resumen - En este trabajo se analiza la acidez de la precipitación en la Península Ibérica durante el periodo 1986-1997. Los principales objetivos son analizar la variabilidad, estacionalidad y tendencia del $\mathrm{pH}$. Los resultados obtenidos muestran que la acidez de la precipitación es mayor en Portugal que en España, aunque en ambos países se registran valores de $\mathrm{pH}$ claramente ácidos. En general, la variabilidad del pH se caracteriza por ser menor en Portugal. También se detecta en ambos países un ciclo estacional con un mínimo claramente marcado en verano y un máximo en invierno. El análisis de tendencia muestra una evolución del pH creciente y significativa en la Península Ibérica, a excepción de la estación de Noia donde la tendencia observada es decreciente.
\end{abstract}

Palabras Clave: precipitación ácida, pH, EMEP, Portugal, España, tendencia.

Abstract - Acidity of PRecipitation in The Iberian Peninsula - Acidity of precipitation in the Iberian Peninsula between 1986 and 1997 is studied in this paper. The main objectives are the analysis of its variability, seasonal cycle, and trend. The results show a higher acidity of precipitation in Portugal than in Spain, although precipitation is acid in both countries. In general, the $\mathrm{pH}$ variability is lower in Portugal than in Spain. In both countries there is a seasonal cycle with a clear minimum in summer and a maximum in winter. The trend analysis shows a $\mathrm{pH}$ that is increasing in Portugal and Spain, except at Noia station where the observed trend is decreasing.

Key words: acid precipitation, pH, EMEP, Portugal, Spain, trend.

Resumo-AnÁlise da ACIDEZ dA PRECIPITAÇão na Península IbÉRICA - Analisa-se a acidez da precipitação na Península Ibérica, durante o período 1986-1997. Os principais objectivos são o estudo da variabilidade e da tendência do $\mathrm{pH}$ da precipitação. Os resultados mostram que a acidez da precipitação é mais acentuada nos locais de medição portugueses, embora, em ambos os países, se registem

\footnotetext{
1 Departamento de Física del Aire, Universidad Complutense, Ciudad Universitaria 28040 Madrid SPAIN. E-mail: eduardo@6000aire.fis.ucm.es Departamento de Física del Aire, Universidad Complutense, Ciudad Universitaria 28040 Madrid ESPAÑA. E-mail: eduardo@6000aire.fis.ucm.es

2 Fraternidad-Muprespa, c/ José Abascal, 50, 28003 Madrid ESPAÑA, Tlf: 3491 3956742, Fax: 3491 3993707. E-mail: nlavin@fraternidad.com

3 Departamento de Métodos Cuantitativos, Universidad de Comillas, c/ Alberto Aguilera, 23, 28015 Madrid ESPAÑA.
} 
precipitações ácidas. A variabilidade do pH é menor em Portugal. Em ambos os Países, detecta-se um ciclo estacional com um mínimo claramente marcado no Verão e um máximo no Inverno. A análise de tendência revela uma evolução crescente do $\mathrm{pH}$ na Península Ibérica, à excepção do posto de observação de Nóia.

Palavras-chave: precipitação ácida, pH, EMEP, Portugal, Espanha, tendência.

\section{INTRODUCCIÓN}

La calidad del aire es uno de los problemas medioambientales que mayor atención ha despertado en las últimas décadas por las consecuencias que tiene sobre la salud de los seres vivos y el entorno (BURTRAw et al., 1997). Así la emisión por el hombre de compuestos químicos en cantidades superiores a las naturales genera innumerables problemas medioambientales. A pesar de que todos ellos son de indudable importancia, no todos generan la misma preocupación gubernamental, social o científica. Uno de los que más atención ha recibido en los últimos años, por su impacto económico y porque las consecuencias del problema no se manifiestan necesariamente en los mismos países donde se genera, es la precipitación o lluvia ácida (EEA, 1998). Este problema no es nuevo, así en el trabajo de Cowling en 1982 se cita el periodo de mediados del siglo XVII como la primera referencia al problema de la precipitación ácida. Aunque el término «lluvia ácida» no aparece hasta 1872 en el libro "Air and Rain» por Angus Smith. Sin embargo, durante el siglo XX el gran desarrollo industrial ha provocado que la contaminación atmosférica y en particular la lluvia ácida se convierta en un serio problema medioambiental y de salud pública. Por ello desde 1950 se han publicado numerosos trabajos sobre el tema. Uno de los más importantes se debe a Rossby y Egnér en 1955. En dicho trabajo se sugiere que la precipitación ácida es un problema regional, siendo los primeros en relacionar procedencias de masas de aire con concentraciones de especies químicas; sugiriendo que elevadas concentraciones de azufre en Suecia se correspondían con trayectorias procedentes del interior del continente europeo.

El grado de acidez de la precipitación se expresa en unidades de $\mathrm{pH}$. La escala de $\mathrm{pH}$ se extiende de 0 a 14 unidades. El agua de lluvia no contaminada tiene un $\mathrm{pH}$ de 5.6; que es el mismo valor que corresponde al agua destilada en equilibrio con el dióxido de carbono (330 ppm) en condiciones normales. Luego el agua de lluvia es ligeramente ácida debido al ácido carbónico, formado cuando el dióxido de carbono atmosférico reacciona con el vapor de agua. Por tanto, se considera situación de precipitación ácida aquella cuyo $\mathrm{pH}$ es inferior a 5.6. El responsable de la acidez de la precipitación es la contaminación producida por la emisión a la atmósfera de cantidades masivas de contaminantes desde las centrales térmicas, automóviles, fundiciones, etc. Las emisiones de dióxido de azufre y de óxidos de nitrógeno, consideradas hasta 
hace poco como problemas locales de contaminación, se sabe actualmente que viajan cientos de kilómetros a través de la atmósfera, se transforman químicamente durante el trayecto y eventualmente retornan a la superficie terrestre en forma de ácidos. Así, cuando esta contaminación no respeta fronteras y su impacto puede estar localizado en países distintos a los países emisores, estamos hablando de contaminación transfronteriza (RÚA, 1996; HERNÁNDEZ, et al., 1995).

El carácter internacional de esta contaminación obliga a adoptar políticas comunes entre los distintos países. Ante esta necesidad se suscribió, en el ámbito de las Naciones Unidas, el Convenio Sobre Transporte a Larga Distancia y Transfronterizo de Contaminantes Atmosféricos. Este Convenio conocido como Convenio de Ginebra entró en vigor en 1983 y fue ratificado por 31 países, entre ellos Portugal y España. Una vez reconocida la importancia de la lluvia ácida, la respuesta investigadora fue inmediata y se establecen redes para medir la composición de la precipitación. En los EEUU surge la NAPAP (National Acid Precipitation Assessment Program) y paralelamente surge en Europa con similares propósitos, la red EMEP (European Monitoring and Evaluation Programme), cuyos datos y estaciones de medida en Portugal y España sirven de base para este trabajo.

En el presente estudio se analizan los valores de pH en la Península Ibérica. El objetivo fundamental es describir el comportamiento temporal del $\mathrm{pH}$ en la Península Ibérica durante el periodo 1986-1997.

\section{DATOS}

Las estaciones analizadas son las siguientes: Bragança $\left(41^{\circ} 49^{\prime} \mathrm{N}, 6^{\circ} 46^{\prime} \mathrm{W}\right.$, $691 \mathrm{~m})$, Viana do Castelo $\left(40^{\circ} 25^{\prime} \mathrm{N}, 7^{\circ} 33^{\prime} \mathrm{W}, 16 \mathrm{~m}\right)$, Monte Velho $\left(38^{\circ} 05^{\prime} \mathrm{N}, 8^{\circ}\right.$ $\left.48^{\prime} \mathrm{W}, 43 \mathrm{~m}\right)$ para Portugal y Toledo $\left(39^{\circ} 33^{\prime} \mathrm{N}, 4^{\circ} 21^{\prime} \mathrm{W}, 515 \mathrm{~m}\right)$, La Cartuja ( $37^{\circ}$ $\left.12^{\prime} \mathrm{N}, 3^{\circ} 36^{\prime} \mathrm{W}, 720 \mathrm{~m}\right)$, Roquetas $\left(40^{\circ} 49^{\prime} \mathrm{N}, 0^{\circ} 30^{\prime} \mathrm{W}, 50 \mathrm{~m}\right)$, Logroño $\left(42^{\circ} 27^{\prime} \mathrm{N}\right.$, $\left.2^{\circ} 21^{\prime} \mathrm{W}, 370 \mathrm{~m}\right)$, Noia $\left(42^{\circ} 44^{\prime} \mathrm{N}, 8^{\circ} 55^{\prime} \mathrm{W}, 685 \mathrm{~m}\right)$ para España. En la figura 1 se puede ver la localización geográfica de dichas estaciones. Estas estaciones forman parte de la Red de Vigilancia de la Contaminación Atmosférica a Gran Distancia, del programa EMEP (European Monitoring Evaluation Programme). Las estaciones EMEP miden contaminación transfronteriza y por tanto evalúan la contaminación de fondo o regional. Los requisitos que deben de cumplir estas estaciones son los siguientes:

- Tienen que estar situadas en ambientes rurales, evitando los ambientes suburbanos, que en un futuro pudieran ser urbanizados.

- Tienen que estar lo suficientemente alejadas de zonas edificadas, con el fin de evitar efectos procedentes de fuentes de contaminación local.

- Tienen que estar ubicadas al menos a $40 \mathrm{Km}$ de distancia de grandes fuentes de contaminación. 
106

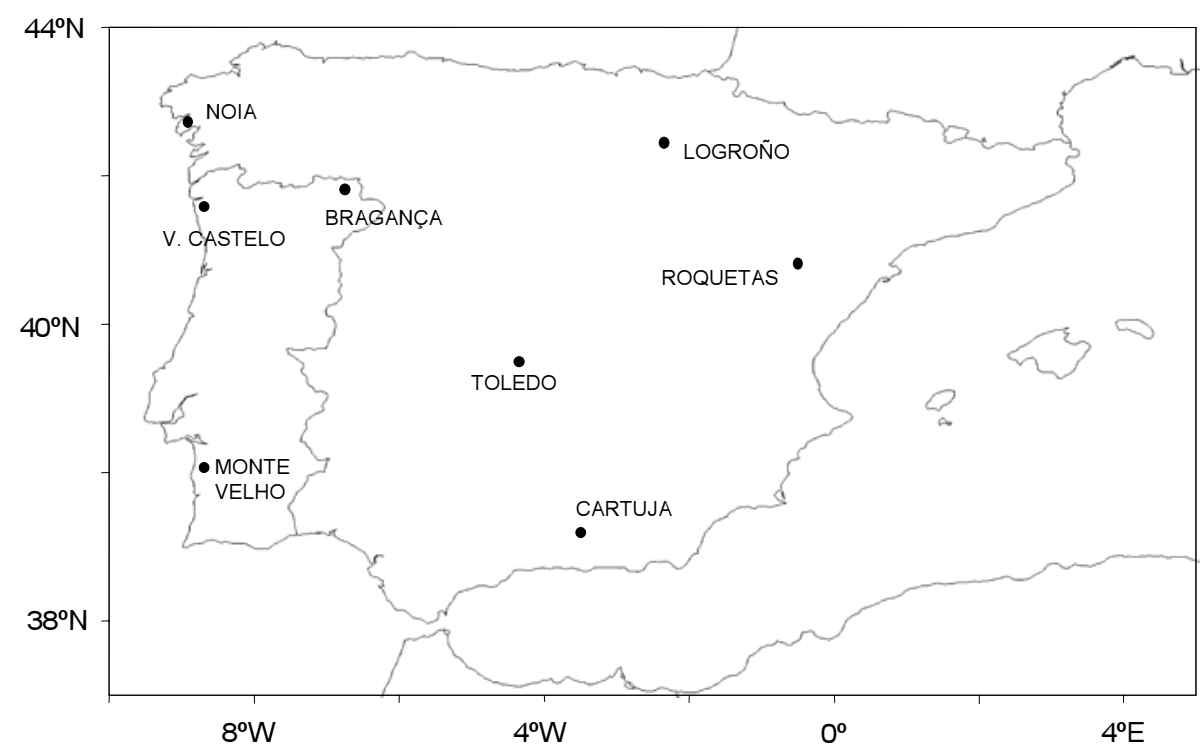

FIG. 1 - Localización geográfica de las estaciones

FIG. 1 -Geographical location of the sites

- Tienen que evitarse los valles y los picos de las montañas.

- Tienen que evitarse ubicaciones donde el viento sea fuerte, siendo el emplazamiento idóneo un claro en el bosque.

En estas estaciones, de acuerdo con el Órgano Director del EMEP, se efectúan medidas de $\mathrm{pH}$, conductividad, sulfato $\left(\mathrm{SO}_{4}^{2-}\right)$, nitrato $\left(\mathrm{NO}_{3}^{-}\right)$, amonio $\left(\mathrm{NH}_{4}^{+}\right)$, calcio $\left(\mathrm{Ca}^{2+}\right)$, potasio $\left(\mathrm{K}^{+}\right)$, cloro $\left(\mathrm{Cl}^{-}\right)$, sodio $\left(\mathrm{Na}^{+}\right)$y magnesio $\left(\mathrm{Mg}^{2+}\right)$ en la precipitación. Los métodos analíticos empleados para determinar la composición de la precipitación se listan en la Tabla I. Las concentraciones de sulfato corresponden con las concentraciones no marinas, es decir, las concentraciones de sulfato total menos aquellas de procedencia marina. La corrección marina es necesario tenerla en cuenta debido a que en las regiones costeras parte de la concentración de sulfato registrada es debido al efecto marino. Los detalles de la red, con los periodos de muestreo y los métodos analíticos se encuentran en LöVBLAND et al. (1995).

En este trabajo se han analizado los valores mensuales del $\mathrm{pH}$ en la precipitación durante el periodo 1986-1997. 
TABLA I - Métodos analíticos empleados para determinar la composición de la precipitación

TABLE I-Analytical methods used to determine the composition of rainfall

\begin{tabular}{|l|c|c|c|}
\hline \multirow{2}{*}{ Ión } & \multirow{2}{*}{ Unidad } & \multicolumn{2}{c|}{ Métodos Analíticos } \\
\cline { 3 - 4 } & & Portugal & España \\
\hline Sulfato $\left(\mathrm{SO}_{4}{ }^{2-}\right)$ & $\mathrm{mgS} / \mathrm{l}$ & Thorin & Cromatografía iónica \\
\hline Nitrato $\left(\mathrm{NO}_{3}^{-}\right)$ & $\mathrm{mgN} / \mathrm{l}$ & Espectrofotometría & Cromatografía iónica \\
\hline Amonio $\left(\mathrm{NH}_{4}^{+}\right)$ & $\mathrm{mg} / \mathrm{l}$ & Espectrofotometría & Espectrofotometría \\
\hline Calcio $\left(\mathrm{Ca}^{2+}\right)$ & $\mathrm{mg} / \mathrm{l}$ & Absorción atómica & Absorción atómica \\
\hline Potasio $\left(\mathrm{K}^{+}\right)$ & $\mathrm{mg} / \mathrm{l}$ & Emisión atómica & Absorción atómica \\
\hline Cloro $\left(\mathrm{Cl}^{-}\right)$ & $\mathrm{mg} / \mathrm{l}$ & Espectrofotometría & Absorción atómica \\
\hline Sodio $\left(\mathrm{Na}^{+}\right)$ & $\mathrm{mg} / \mathrm{l}$ & Absorción atómica & Absorción atómica \\
\hline Magnesio $\left(\mathrm{Mg}^{2+}\right)$ & $\mathrm{mg} / \mathrm{l}$ & Absorción atómica & Cromatografía iónica \\
\hline
\end{tabular}

\section{ANÁLISIS TEMPORAL}

\section{Análisis estadístico del pH para la Península Ibérica}

En este apartado llevamos a cabo una descripción estadística de los valores del pH para el periodo de estudio 1986-1997. Asimismo y cara a observar la diferente dispersión de los datos, nada mejor que utilizar como medida el coeficiente de variación (CV), que es una medida relativa de variabilidad. El coeficiente de variación se define como el cociente entre la desviación típica y la media de la serie de estudio. Este coeficiente da idea de cuantas veces la media aritmética está contenida en la desviación típica, de modo que, cuanto más lejos esté de cero, mayor dispersión presentará la muestra (RÚA y MARín, 1999).

En la Tabla II se resumen algunas de las medidas de posición y dispersión más interesantes, que sirven para sintetizar el comportamiento del pH. En dicha tabla recogemos el número de observaciones (N); media aritmética (Media), desviación típica (Des. Típ.), coeficiente de variación en \% (CV), valor mínimo, valor máximo (Máx.) y rango.

El análisis de los estadísticos de la Tabla II muestra unos valores medios de pH superiores en España. Esto indica que la precipitación caída en España es menos ácida que la caída en Portugal. En las estaciones de Viana de Castelo y Monte Velho se registran valores medios ligeramente ácidos (en torno a 5.4). En el resto de Europa los valores medios de $\mathrm{pH}$ son generalmente inferiores a 5.0, siendo Centro-Europa donde se registran los valores medios más bajos as mayores emisiones de contaminantes causantes de la precipitación ácida 
TABLA II - Estadísticos descriptivos para la precipitación en las estaciones peninsulares, 1986-1997

TABLE II - Statistics for precipitation, for peninsular sites, 1986-1997

\begin{tabular}{|l|c|c|c|c|c|c|c|}
\hline \multicolumn{1}{|c|}{ Estación } & N & Media & Des. Típ. & CV (\%) & Mínimo & Máx. & Rango \\
\hline Bragança & 101 & 5.6 & 0.38 & 6 & 4.1 & 6.7 & 2.6 \\
\hline V. Castelo & 107 & 5.3 & 0.37 & 7 & 4.3 & 6.7 & 2.4 \\
\hline Monte Velho & 107 & 5.5 & 0.62 & 11 & 4.4 & 6.9 & 2.5 \\
\hline Toledo & 132 & 6.0 & 0.60 & 10 & 4.5 & 6.5 & 4.0 \\
\hline Cartuja & 125 & 6.7 & 0.80 & 12 & 3.5 & 7.9 & 4.4 \\
\hline Roquetas & 126 & 6.6 & 0.56 & 8 & 4.5 & 7.9 & 3.4 \\
\hline Logroño & 133 & 6.3 & 0.53 & 8 & 4.0 & 7.4 & 3.4 \\
\hline Noia & 101 & 5.5 & 0.57 & 10 & 4.3 & 7.0 & 2.7 \\
\hline
\end{tabular}

(PuXBaum et al. 1998) debido a l(EMEP, 1999), mientras que en el sur de Europa se registran los mayores valores medios de $\mathrm{pH}$.

No obstante, los mínimos registrados en ambos países son claramente ácidos, con valores de $\mathrm{pH}$ inferiores a 5.6. Así en Portugal se observan mínimos en torno a 4.4 y en España ligeramente inferiores a los portugueses en torno a 4.2. El mínimo más acusado para toda la Península Ibérica es de 3.5 y se dio en la estación de Toledo en agosto de 1993. En Portugal el mínimo alcanzado durante todo el periodo de estudio fue de 4.3 en Viana de Castelo, en junio de 1989. Por otro lado, los máximos registrados en España tienen valores superiores a 7.0, mientras que en Portugal los máximos no superan los 6.9. El máximo para toda la Península Ibérica se observó en la estación de Roquetas con un valor de 7.9 en marzo de 1992. En Portugal el máximo registro fue de 6.9 en Monte Velho, en marzo de 1995.

Por otra parte se observa que la variabilidad del pH es menor en Portugal que en España, como se desprende del resultado obtenido para el coeficiente de variación (CV) y el rango. Así el CV es aproximadamente un $10 \%$ menor en Portugal que en España. No obstante, la estación de Monte Velho muestra una alta variabilidad en comparación con el resto de estaciones portuguesas. De hecho la variabilidad en Monte Velho es aproximadamente un 35\% superior a la del resto de estaciones situadas en Portugal. También es importante mencionar el comportamiento semejante observado entre las estaciones de Monte Velho y Noia. Esto se justifica por la situación de dichas estaciones. De hecho ambas estaciones son costeras y se encuentran alejadas de las fuentes emisoras a distancias muy similares.

Un hecho relevante es que los mínimos observados en las distintas estaciones corresponden con los meses de verano, mientras que los máximos corres- 
ponden con los meses de otoño e invierno. Por esta razón en el siguiente apartado se realiza un análisis estacional.

\section{Variabilidad estacional del pH}

Puesto que la radiación juega un papel importante en la reacción de los contaminantes que producen la lluvia ácida (WARNECK, 1988), nos ha parecido oportuno efectuar un análisis estacional. Por lo tanto, se analizan los valores del pH para las cuatro estaciones del año: invierno (enero, febrero, marzo), primavera (abril, mayo, junio), verano (julio, agosto, septiembre) y otoño (octubre, noviembre, diciembre).

En la figura 2 se muestra la variación estacional en Bragança y Toledo. Ambas estaciones reflejan el comportamiento estacional del pH en la Península Ibérica.

En general se observa que durante el verano se alcanzan los valores más bajos para el $\mathrm{pH}$. Este resultado es debido a que durante el verano la insolación es máxima (INM, 1988), favoreciéndose la formación de compuestos ácidos, principalmente por oxidación del $\mathrm{SO}_{2}$ (RÚA et al., 1998). Por ello la precipitación caída durante el verano es más ácida que la del resto del año. No obstante las consecuencias son pequeñas debido a que la precipitación caída durante el verano es inferior a la del invierno. En concreto, para el verano en Portugal, la precipitación media es de $13.7 \mathrm{~mm}$ y en España es de $26.3 \mathrm{~mm}$, mientras que en invierno es de $78.7 \mathrm{~mm}$ y $81.3 \mathrm{~mm}$ en Portugal y España respectivamente durante el periodo de estudio. Durante el invierno sucede el efecto inverso, es decir, la radiación solar recibida es menor, por lo que suele ser mayor el pH de la precipitación. Estos resultados están en concordancia con los máximos encontrados para el SO ${ }_{4}^{2-}$ en España (RÚA et al., 1998), y los hallados en el noroeste de los Estados Unidos (TERRY y EASTER, 1987).

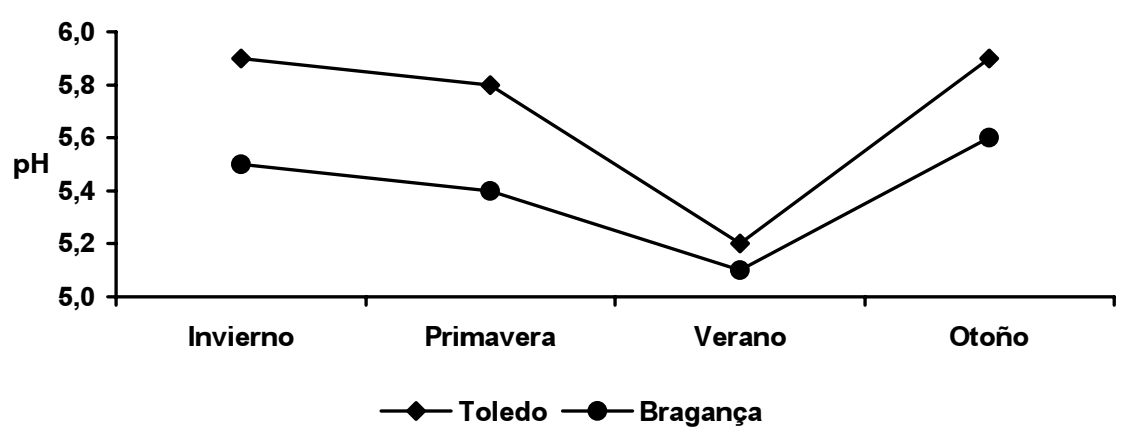

FIg. 2 - Variación del pH estacional medio en Bragança y Toledo (1986-1997)

FIG. 2 - Seasonal average of pH in Bragança and Toledo (1986-1997) 


\section{Análisis de tendencia}

En este apartado se lleva a cabo un análisis de tendencia y estacional del pH para cada una de las estaciones consideradas en este estudio. Para realizar dicho análisis se ha utilizado la serie completa de valores mensuales medios desde 1986 hasta 1997.

Para conocer si existe tendencia o variación estacional se usa el siguiente modelo de regresión (GIMENO y HERNÁNDEZ, 1997):

$$
y=a+b i+d \cos \left(2 \pi t-\Phi+e_{i}\right)
$$

donde y es la variable dependiente que representa el valor del pH para el mes i, el término que contiene el coseno representa una variación cíclica anual de amplitud d y de fase $\Phi$. En este término la t representa el tiempo en años que es calculado para cada mes i como $t=i / 12(i=1, \ldots, 144)$. El coeficiente b representa la tendencia lineal y por último $\mathrm{e}_{\mathrm{i}}$ es el término error o residual del modelo que sigue una distribución normal.

Para calcular los parámetros de la ecuación 1, el modelo se escribe de la siguiente forma:

$$
y=a+b t+d_{1} \cos \left(2 \pi t+d_{2} \sin (2 \pi t)+e_{i}\right.
$$

donde $\mathrm{d}_{1}=\operatorname{d} \cos \Phi \mathrm{y} \mathrm{d}_{2}=\mathrm{d} \sin \Phi$.

Los valores estimados para los parámetros de la ecuación 2, su significación y el valor de $\mathrm{R}^{2}$ se muestran en la Tabla III.

TABLA III - Estimadores obtenidos para el modelo de regresión empleado TABLE III - Estimators obtained for the regression model

\begin{tabular}{|l|c|c|c|c|}
\hline \multicolumn{1}{|c|}{ Estación } & $\mathbf{b}\left(\mathbf{p H}\right.$ mes $\left.^{-1}\right)$ & $\mathbf{d}_{\mathbf{1}}$ & $\mathbf{d}_{\mathbf{2}}$ & $\mathbf{R}^{\mathbf{2}}$ \\
\hline Bragança & $+0.002^{*}$ & 0.023 & 0.015 & 0.40 \\
\hline V. Castelo & $+0.002^{*}$ & 0.015 & $0.16^{*}$ & 0.39 \\
\hline Monte Velho & $+0.003^{*}$ & 0.010 & 0.011 & 0.15 \\
\hline Toledo & $+0.0001^{*}$ & 0.017 & 0.09 & 0.10 \\
\hline Cartuja & $+0.001^{*}$ & 0.021 & $0.18^{*}$ & 0.20 \\
\hline Roquetas & $+0.002^{*}$ & 0.015 & 0.01 & 0.21 \\
\hline Logroño & $+0.005^{*}$ & 0.011 & $0.17^{*}$ & 0.23 \\
\hline Noia & $-0.009^{*}$ & 0.013 & $0.15^{*}$ & 0.22 \\
\hline
\end{tabular}

* Significativo al nivel de 0.05 
Los resultados obtenidos muestran que existe una tendencia creciente y significativa del pH en la Península, salvo en la estación de Noia que muestra el comportamiento contrario. Los valores para las pendientes positivas se encuentran entre $+0.005 \mathrm{pH}^{-1} \mathrm{mes}^{-1} \mathrm{y}+0.0001 \mathrm{pH}^{\mathrm{mes}}{ }^{-1}$. También se observa una oscilación anual significativa asociada al término del seno para las estaciones de V. Castelo, Cartuja, Logroño y Noia. Por último mencionar que el ajuste del modelo propuesto tiene un valor $\mathrm{R}^{2}$ en torno a 0.25 para el conjunto de estaciones analizadas, significativo a un nivel de 0.10 .

\section{RESUMEN Y CONCLUSIONES}

En el presente trabajo se ha efectuado un análisis del comportamiento temporal, variabilidad y tendencia del $\mathrm{pH}$ para la Península Ibérica durante el periodo 1986-1997. La importancia del pH es indudable, ya que determina la acidez de la precipitación y sus consecuencias son del todo significativas sobre el medioambiente.

Después de este estudio y a modo de resumen cabe destacar las siguientes conclusiones:

* Los valores medios del pH en la Península Ibérica están en torno a 5.8, siendo los registros observados en España superiores a los registrados en Portugal. Este resultado se debe a que las emisiones de $\mathrm{SO}_{2}$ (uno de los principales contaminantes responsables de la lluvia ácida) han descendido en España más que en Portugal (Lavín y MARín, 2000). No obstante, es importante destacar la existencia de mínimos claramente por debajo del límite de 5.6 en ambos países.

* La evolución estacional del pH se caracteriza por alcanzar los mínimos durante el verano y los máximos en el invierno.

* El análisis de tendencia muestra una evolución creciente y significativa en todas las estaciones analizadas, excepto en Noia. Esto indica que la evolución del pH con el tiempo es creciente. Este resultado esta claramente asociado con las políticas de ambos países de reducción de las emisiones de contaminantes (UN/ECE, 1998). La tendencia decreciente observada en Noia se justifica por la fuerte emisión local de contaminantes procedentes principalmente de la región gallega. No osbtante, el valor de la pendiente, aunque negativo, tiene un módulo pequeño lo que nos indica que el descenso es muy suave. 


\section{AGRADECIMIENTOS}

Los autores quieren expresar su agradecimiento a Anne Hjellbrekke por proporcionarnos los datos usados en este trabajo, a Maite Saldaña por su colaboración y a dos revisores anónimos cuyos comentarios han mejorado el manuscrito original.

\section{BIBLIOGRAFÍA}

Burtraw, D.; A. Krupnick; Mansur; E D. Austin; D. Farrell (1997) - The costs and benefits of reducing acid rain. Resources of the Future. Washington.

Cowling, E. (1982) - Acid precipitation in historical perspective. Environmental Science and Technology, 16: 110-123.

EmeP (1999) $-\mathrm{SO}_{2}$ and $\mathrm{NO}_{x}$ emission inventories. Meteorological Synthesizing Centre-West (MSC-W).

EUROPEAN ENVIRONMENTAL AGENCY, EEA (1998)-Europe's environment: the second assessment. Copenhagen, Denmark.

Gimeno, L.; E. Hernández (1997) - The acidifying potential of atmospheric wed deposition in Spain. Toxicological and Environmental Chemistry, 59: 209-217.

Hernández, E.; A. Rúa; J. De Las Parras (1995) - Precipitación ácida en la estación EMEP de Noia. Resúmenes de comunicaciones. XXV Reunión Bienal Real Sociedad Española de Física, A3: 37-40.

INSTITUTO NACIONAL DE METEOROLOGÍA (1988) - Guía resumida del clima en España 1961-1990. Ed. INM, Ministerio de Medio Ambiente.

LaVín, N.; E. Marín (2000) - Trends of $\mathrm{SO}_{2}$ emission in the European Union, 1985-1997. Toxicological and Environmental Chemistry, (in press).

LövBlAND, G. et al. (1995)-EMEP/CCC-Rep 2/95 Norwegian Institute for Air Research, Lillestron.

Puxbaum, H.; V. Simeonov; F. Kalina (1998) - Ten years trends (1984-1993) in the precipitation chemistry in central Austria. Atmospheric Environment, 2: 193-202.

Rossby, C.; H. EgnéR (1955) - On the chemical climate and its variation with the atmospheric circulation pattern. Tellus, 7: 118-133.

RÚA, A. (1996) - Contaminación atmosférica transfronteriza: Análisis Espacio-Temporal, Fuentes y Modelización. Tesis Doctoral, Universidad Complutense Madrid.

RÚA, A.; E. MARín (1999) - Análisis de la variabilidad y tendencia climática en Ourense. Boletin Auriense, XXVIII: 229-249.

RÚA, A.; L. Gimeno; HeRnández E. (1998) - Trends and seasonal variation of $\mathrm{SO}_{2}, \mathrm{NO}_{\mathrm{x}}, \mathrm{SO}_{4}^{2-}$ and $\mathrm{NO}_{3}^{-}$concentrations in the air of the spanish EMEP stations. Toxicological and Environmental Chemistry, 65: 153-161. 
TERRY, M.; C. EASTER (1987) - Statistical summary and analyses of event precipitation chemistry from the MAP3S network, 1976-1983. Atmospheric Environment, 21(1): 113-128.

UNITED NATIONS/ECONOMIC COMMISSION EUROPE, UN/ECE (1998) - The convention on Long-range Transboundary Air Pollution. Denmark.

WARNECK, T. (1988) - Chemistry of the natural atmosphere. International Geophysics Series, Academic Press, Inc. 